\section{A Publicly Funded Clinical Trials Network: Do We Need It?}

I'm worried. Really worried. As I look at the clinical trial activity at our own UCSF Helen Diller Family Comprehensive Cancer Center and hear the stories of other centers around the country, I feel like we are losing ground. Not in scientific excellence, mind you, but in execution. We have great ideas. But launching investigator-initiated trials with the hope of industry sponsorship or conducting trials in the US Clinical Trials Cooperative Group Program (as of March 1, 2014, called the National Clinical Trials Network [NCTN]) is getting harder and harder. Securing funding for the trials is difficult, and when funding is available, it usually doesn't come close to the true cost. Many-maybe most-institutions do not have the financial bandwidth to subsidize these studies.

I'm particularly worried about the NCTN itself. For years, the various cooperative groups functioned unevenly and inefficiently. This was highlighted in 2010 by an Institute of Medicine (IOM) report entitled, "A National Cancer Clinical Trials System for the 21st Century: Reinvigorating the NCI Cooperative Group Program,"1 which evaluated the quality and productivity of the cooperative group effort. It wasn't a very good review. In fact, the committee called for a complete revamping of the cooperative group effort. Importantly, the report affirmed the importance of our cooperative groups and the need to improve the system.

Unfortunately, though, reforming a system that was historically based on a culture of independence and competitiveness is not easy. The NCI responded to the IOM report by creating a new entity, the $\mathrm{NCTN},{ }^{2}$ which collapsed the 10 existing cooperative groups into 4 adult groups and 1 pediatric group and changed the funding model. As part of this new system, 30 academic institutions, called Lead Academic Participating Sites, will receive additional funding to support infrastructure needs. However, institutions that didn't compete or competed and lost are apparently out of luck, as these grants aren't expected to be offered again for some time. Rumor has it that the NCI is looking for additional costcutting measures (who isn't?) and is addressing this through consolidating common core resources within the network, such as biostatistics support.

I'm not suggesting that any of these moves were or are unwise. I'm just noting that all of these changes have had a significant impact on productivity. The timeline from idea to implementation has slowed considerably. Accrual to open studies is often below target. Investigators with fresh ideas are frustrated. And industry, for the most part, would rather conduct its own studies.

Fundamentally, well-designed and flawlessly conducted clinical trials that ask important questions are at the core of evidence-based medicine. In fact, I would argue that clinical trials are largely responsible for the dramatic decline seen in death rates from some of the most common cancers. And the US Clinical Trials Cooperative Group Program has played an enormous role in conducting landmark studies, such as those that defined adjuvant therapy in breast and colorectal cancers, two of the biggest success stories.

So my answer to the title question is "yes," we need a publicly funded clinical trials network. The pharmaceutical industry should and will continue to conduct trials with patented drugs for initial and subsequent expanded indications. We need that too. But we also need a forum in which to test ideas when there is no sponsor. We belong to a robust scientific community with many important ideas (involving drugs, devices, assessment of biological behavior, and so forth) that are worthy of our attention. We need to monitor the changes in the system and make sure that our public clinical trials network is efficient and productive. Future generations will be better for it.

\section{References}

1. Nass SJ, Moses HL, Mendelson J, eds. A National Cancer Clinical Trials System for the 21ST Century: Reinvigorating the NCI Cooperative Group Program. Washington, DC: Institute of Medicine; 2010.

2. National Cancer Institute. An overview of NCI's National Clinical Trials Network. Washington, DC: National Institutes of Health; 2014.

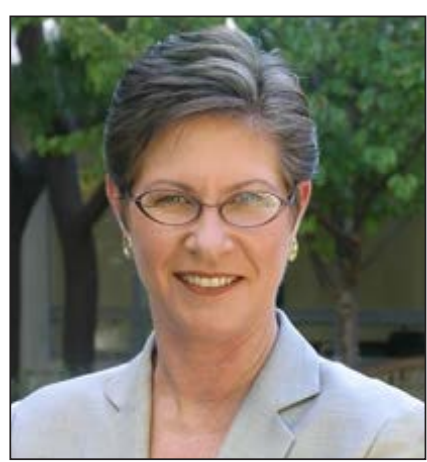

Margaret Tempero, MD

Dr. Tempero is a Professor of Medicine and Director of the UCSF Pancreas Center, and the editor-in-chief of JNCCN. Her research career has focused on pancreatic ductal adenocarcinoma especially in the area of investigational therapeutics. Dr. Tempero has served on the ASCO Board of Directors and as ASCO President. She co-directed the AACR/ASCO Methods in Clinical Cancer Research and taught this course and similar courses in Europe and Australia. She was founding Chair of the $\mathrm{NCl}$ Clinical Oncology Study Section (CONC) and served as a member and chair of the $\mathrm{NCl}$ Board of Scientific Counselors Subcommittee A. She is on the External Advisory Boards of the Pancreas SPOREs at Mayo Clinic and at $\mathrm{UAB} /$ Minnesota and the GI SPORE at the University of Arizona. She is, or has been, on the Scientific Advisory Boards of the Lustgarten Foundation, the Pancreatic Cancer Action Network, the V Foundation, The Alberta Canada Cancer Board, and the EORTC. She served as a member of the Oncology Drug Advisory Committee for the FDA.

She has served as Deputy Director and Interim Director for the UNMC Eppley Cancer Center. She is Chief Emeritus of the Division of Medical Oncology at UCSF and served as Deputy Director and Director of Research Programs at the UCSF Helen Diller Family Comprehensive Cancer Center.

The ideas and viewpoints expressed in this editorial are those of the author and do not necessarily represent any policy, position, or program of NCCN. 